\title{
Clover improvement for stout-smeared $2+1$ flavour SLiNC fermions: perturbative results
}

\section{R. Horsley}

School of Physics and Astronomy, University of Edinburgh, Edinburgh EH9 3JZ, UK

E-mail: rhorsleyeph.ed.ac.uk

\section{H. Perlt*}

Institut für Theoretische Physik, Universität Leipzig, D-04109 Leipzig, Germany

E-mail: perlteitp.uni-leipzig.de

\section{P.E.L. Rakow}

Theoretical Physics Division, Department of Mathematical Sciences, University of Liverpool, Liverpool L69 3BX, UK

E-mail: rakow@amtp.liv.ac.uk

\section{G. Schierholz}

Deutsches Elektronen-Synchrotron DESY, D-22603 Hamburg, Germany

E-mail: Gerrit.Schierholzedesy.de

\section{A. Schiller}

Institut für Theoretische Physik, Universität Leipzig, D-04109 Leipzig, Germany

E-mail: schillereitp.uni-leipzig.de

\section{(QCDSF collaboration)}

For the Stout Link Non-perturbative Clover (SLiNC) action we determine in one-loop lattice perturbation theory the critical hopping parameter $\kappa_{c}$ and the clover parameter $c_{S W}$ which is needed for $\mathscr{O}(a)$ improvement. Performing this calculation off-shell we are also able to compute the non gauge invariant quark field improvement coefficient $c_{N G I}$. Additionally, we present first results for the renormalization factors of the scalar, pseudoscalar, vector and axial vector currents. We discuss mean field improvement for the SLiNC action.

The XXVI International Symposium on Lattice Field Theory

July 14-19 2008

Williamsburg, Virginia, USA

\footnotetext{
* Speaker.
} 


\section{Introduction}

Simulations of Wilson-type fermions at realistic quark masses require an improved action with good chiral properties and scaling behavior. A systematic improvement scheme that removes discretization errors order by order in the lattice spacing $a$ has been proposed by Symanzik [1] and developed for on-shell quantities in [2,3]. $\mathscr{O}(a)$ improvement of the Wilson fermion action is achieved by complementing it with the so-called clover term [3], provided the associated clover coefficient is tuned properly.

The focus of this contribution is to determine the clover coefficient and the additive mass renormalization for plaquette and Symanzik improved gauge action and stout link clover fermions in one-loop lattice perturbation theory. We correct earlier results published in [4] and introduce a modified mean field improvement for partially smeared links. A detailed discussion can be found in [5]. Additionally, in this paper we present first results for the one-loop renormalization factors of the scalar, pseudoscalar, vector and axial vector currents with the chosen action. First nonperturbative results obtained with this action are presented in [6].

The Symanzik improved gauge action reads [1]

$$
S_{G}^{\text {Sym }}=\frac{6}{g^{2}}\left\{c_{0} \sum_{\text {Plaquette }} \frac{1}{3} \operatorname{Re} \operatorname{Tr}\left(1-U_{\text {Plaquette }}\right)+c_{1} \sum_{\text {Rectangle }} \frac{1}{3} \operatorname{Re} \operatorname{Tr}\left(1-U_{\text {Rectangle }}\right)\right\}
$$

with $c_{0}+8 c_{1}=1$ and

$$
c_{0}=\frac{5}{3}, \quad c_{1}=-\frac{1}{12} .
$$

Clover fermions have the action for each quark flavor [3]

$$
\begin{aligned}
S_{F}= & a^{4} \sum_{x}\left\{-\frac{1}{2 a}\left[\bar{\psi}(x) \widetilde{U}_{\mu}(x)\left(1-\gamma_{\mu}\right) \psi(x+a \hat{\mu})+\bar{\psi}(x) \widetilde{U}_{\mu}^{\dagger}(x-a \hat{\mu})\left(1+\gamma_{\mu}\right) \psi(x-a \hat{\mu})\right]\right. \\
& \left.+\frac{1}{a}\left(4+a m_{0}+a m\right) \bar{\psi}(x) \psi(x)-c_{S W} g \frac{a}{4} \bar{\psi}(x) \sigma_{\mu \nu} F_{\mu \nu}(x) \psi(x)\right\},
\end{aligned}
$$

where

$$
a m_{0}=\frac{1}{2 \kappa_{c}}-4
$$

$\kappa_{c}$ being the critical hopping parameter, is the additive mass renormalization term, and $F_{\mu v}(x)$ is the field strength tensor in clover form with $\sigma_{\mu v}=(i / 2)\left(\gamma_{\mu} \gamma_{\nu}-\gamma_{v} \gamma_{\mu}\right)$. We consider a version of clover fermions in which we do not smear links in the clover term, but the link variables $U_{\mu}$ in the next neighbor terms have been replaced by (uniterated) stout links [7]

$$
\widetilde{U}_{\mu}(x)=e^{i Q_{\mu}(x)} U_{\mu}(x)
$$

with

$$
Q_{\mu}(x)=\frac{\omega}{2 i}\left[V_{\mu}(x) U_{\mu}^{\dagger}(x)-U_{\mu}(x) V_{\mu}^{\dagger}(x)-\frac{1}{3} \operatorname{Tr}\left(V_{\mu}(x) U_{\mu}^{\dagger}(x)-U_{\mu}(x) V_{\mu}^{\dagger}(x)\right)\right] .
$$

$V_{\mu}(x)$ denotes the sum over all staples associated with the link and $\omega$ is a tunable weight factor. Stout smearing is preferred because (1.5) is expandable as a power series in $g^{2}$, so we can use perturbation theory. Many other forms of smearing do not have this nice property. Because both 
the unit matrix and the $\gamma_{\mu}$ terms are smeared, each link is still a projection operator in the Dirac spin index.

The reason for not smearing the clover term is that we want to keep the physical extent in lattice units of the fermion matrix small which is relevant for non-perturbative calculations. In that respect we refer to these fermions as SLiNC fermions, from the phrase Stout Link Non-perturbative Clover. The improvement coefficient $c_{S W}$ as well as the additive mass renormalization $a_{0}$ are associated with the chiral limit. So we will carry out the calculations for massless quarks, which simplifies things, though it means that we cannot present values for the mass dependent corrections.

In perturbation theory

$$
c_{S W}=1+g^{2} c_{S W}^{(1)}+\mathscr{O}\left(g^{4}\right) .
$$

The one-loop coefficient $c_{S W}^{(1)}$ has been computed for the plaquette action using twisted antiperiodic boundary conditions [8] and Schrödinger functional methods [9]. Moreover, using conventional perturbation theory, Aoki and Kuramashi [10] have computed $c_{S W}^{(1)}$ for certain improved gauge actions. All calculations were performed for non-smeared links and limited to on-shell quantities.

We extend previous calculations of $c_{S W}^{(1)}$ to include stout links. This is done by computing the one-loop correction to the off-shell quark-quark-gluon three-point function. The improvement of the action is not sufficient to remove discretization errors from Green functions. To achieve this, one must also improve the quark fields [5]

$$
\psi_{\star}(x)=\left(1+a c_{D} \overrightarrow{D D}+\operatorname{aig} c_{N G I} A(x)\right) \psi(x),
$$

where the improvement factor $c_{N G I}$ has been introduced by [11] and has the perturbative expansion

$$
c_{N G I}=g^{2} c_{N G I}^{(1)}+\mathscr{O}\left(g^{4}\right) .
$$

A detailed discussion of the implications of off-shell improvement is given in [5]. In this contribution we concentrate on the on-shell relevant parameters $c_{S W}$ and $\kappa_{c}$.

\section{Off-shell improvement}

It is known [10] that the one-loop contribution of the Sheikoleslami-Wohlert coefficient in conventional perturbation theory can be determined using the quark-quark-gluon vertex $\Lambda_{\mu}\left(p_{1}, p_{2}, c_{S W}\right)$ sandwiched between on-shell quark states. $p_{1}\left(p_{2}\right)$ denotes the incoming (outgoing) quark momentum. In general that vertex is an amputated three-point Green function.

Let us look at the $\mathscr{O}(a)$ expansion of tree-level $\Lambda_{\mu}^{(0)}\left(p_{1}, p_{2}, c_{S W}\right)$ which is derived from action (1.3)

$$
\Lambda_{\mu}^{(0)}\left(p_{1}, p_{2}, c_{S W}\right)=-i g \gamma_{\mu}-g \frac{1}{2} a \mathbf{1}\left(p_{1}+p_{2}\right)_{\mu}+c_{S W} i g \frac{1}{2} a \sigma_{\mu \alpha}\left(p_{1}-p_{2}\right)_{\alpha}+\mathscr{O}\left(a^{2}\right) .
$$

It is obvious from (2.1) that a one-loop calculation of the quark-quark-gluon vertex provides the needed relation to compute $c_{S W}$ in one -loop also.

The off-shell improvement condition states that the non-amputated improved quark-quarkgluon Green function $G_{\star \mu}\left(p_{1}, p_{2}, q\right)$ has to be free of $\mathscr{O}(a)$ terms in one-loop accuracy. The relation between the amputated and non-amputated Green functions is

$$
G_{\mu}\left(p_{1}, p_{2}, q\right)=S\left(p_{2}\right) \Lambda_{v}\left(p_{1}, p_{2}, q, c_{S W}^{(1)}\right) S\left(p_{1}\right) K_{v \mu}(q) .
$$


$K_{v \mu}(q)$ denotes the full gluon propagator which is $\mathscr{O}(a)$-improved already, $S(p)$ the corresponding quark propagator. Using the improved quark fields one obtains the following off-shell improvement condition in momentum space (for details of the derivation see [5])

$$
\begin{aligned}
\Lambda_{\mu}\left(p_{1}, p_{2}, q, c_{S W}^{(1)}\right)= & \Lambda_{\star \mu}\left(p_{1}, p_{2}, q\right)+a g^{3} c_{N G I}^{(1)}\left(\not p_{2} \gamma_{\mu}+\gamma_{\mu} \not p_{1}\right) \\
& -\frac{a}{2} i \not p_{2} \frac{\Sigma_{2}\left(p_{2}\right)}{\Sigma_{1}\left(p_{2}\right)} \Lambda_{\star \mu}\left(p_{1}, p_{2}, q\right)-\frac{a}{2} \Lambda_{\star \mu}\left(p_{1}, p_{2}, q\right) i \not p_{1} \frac{\Sigma_{2}\left(p_{1}\right)}{\Sigma_{1}\left(p_{1}\right)},
\end{aligned}
$$

where the improved three-point function $\Lambda_{\star \mu}\left(p_{1}, p_{2}, q\right)$ is free of $\mathscr{O}(a)$ terms. In (2.3) the quantities $\Sigma_{i}(p)$ are the corresponding contributions to the quark self energy

$$
\Sigma(p)=\frac{1}{a} \Sigma_{0}+i \not p \Sigma_{1}(p)+\frac{a p^{2}}{2} \Sigma_{2}(p) .
$$

\section{Results for improvement parameters}

The calculation has been performed in general covariant gauge. We use a combination of symbolic and numeric routines.

The anticipated general structure for the amputated three-point function at one-loop is

$$
\begin{aligned}
\Lambda_{\mu}\left(p_{1}, p_{2}, q\right) & =\Lambda_{\mu}^{\overline{M S}}\left(p_{1}, p_{2}, q\right)+A_{\mathrm{lat}} i \frac{g^{3}}{16 \pi^{2}} \gamma_{\mu} \\
& +B_{\mathrm{lat}} \frac{a}{2} \frac{g^{3}}{16 \pi^{2}}\left(\not p_{2} \gamma_{\mu}+\gamma_{\mu} \not p_{1}\right)+C_{\mathrm{lat}} i \frac{a}{2} \frac{g^{3}}{16 \pi^{2}} \sigma_{\mu \alpha} q_{\alpha}
\end{aligned}
$$

$\Lambda_{\mu}^{\overline{M S}}\left(p_{1}, p_{2}, q\right)$ is the universal part of the three-point function, independent of the chosen gauge action, computed in the $\overline{M S}$-scheme. It is given in a complete symbolic form in [5].

If we insert (3.1) into the off-shell improvement relation (2.3) we get the following conditions that all terms of order $\mathscr{O}\left(a g^{3}\right)$ have to vanish

$$
\begin{aligned}
\left(c_{S W}^{(1)}-\frac{C_{\mathrm{lat}}}{16 \pi^{2}}\right) \sigma_{\mu \alpha} q_{\alpha} & =0, \\
\left(c_{N G I}^{(1)}-\frac{1}{32 \pi^{2}}\left(A_{\mathrm{lat}}-B_{\mathrm{lat}}-\Sigma_{21}\right)\right)\left(\not p_{2} \gamma_{\mu}+\gamma_{\mu} \not \not_{1}\right) & =0,
\end{aligned}
$$

with $\Sigma_{21}$ defined from (2.4) as

$$
\begin{aligned}
\frac{\Sigma_{2}(p)}{\Sigma_{1}(p)} & =1+\frac{g^{2} C_{F}}{16 \pi^{2}}\left((1-\xi)\left(1-\log \left(a^{2} p^{2}\right)\right)+\Sigma_{21,0}\right) \\
& \equiv 1+\frac{g^{2} C_{F}}{16 \pi^{2}}\left((1-\xi)\left(1-\log \left(p^{2} / \mu^{2}\right)\right)\right)+\frac{g^{2}}{16 \pi^{2}} \Sigma_{21}
\end{aligned}
$$

and

$$
\Sigma_{21}=C_{F}\left(-(1-\xi) \log \left(a^{2} \mu^{2}\right)+\Sigma_{21,0}\right) .
$$

The constant $\Sigma_{21,0}$ depends on the chosen lattice action. Inserting the numbers for the Symanzik action we get the following results for the clover improvement coefficient

$$
\begin{aligned}
c_{S W}^{(1)} & =C_{F}\left(0.116185+0.828129 \omega-2.455080 \omega^{2}\right) \\
& +N_{c}\left(0.013777+0.015905 \omega-0.321899 \omega^{2}\right),
\end{aligned}
$$


and for the off-shell quark field improvement coefficient

$$
c_{N G I}^{(1)}=N_{c}(0.002395-0.010841 \omega) .
$$

For $\omega=0$ the Symanzik result (3.6) agree, within the accuracy of our calculations, with the number quoted in [10].

The additive mass renormalization is given by

$$
a m_{0}=\frac{g^{2} C_{F}}{16 \pi^{2}} \frac{\Sigma_{0}}{4} .
$$

This leads to the critical hopping parameter $\kappa_{c}$, at which chiral symmetry is approximately restored,

$$
\kappa_{c}=\frac{1}{8}\left(1-\frac{g^{2} C_{F}}{16 \pi^{2}} \frac{\Sigma_{0}}{4}\right)
$$

We obtain the following perturbative expression for $\kappa_{c}$

$$
\kappa_{c}=\frac{1}{8}\left[1+g^{2} C_{F}\left(0.037730-0.662090 \omega+2.668543 \omega^{2}\right)\right] .
$$

$a m_{0}$ can be tuned to zero for admissible values of $\omega$. Using the smaller possible value we find $\omega=0.088689$ for the Symanzik gauge action which is not far away from the value $\omega=0.1$ used in our non-perturbative calculations [6].

\section{Mean field improvement}

In the mean field approximation we typically assume that the gauge fields on each link are independently fluctuating variables, and that we can simply represent the links by an average value $u_{0}$. Typical choices for $u_{0}$ would be to choose $u_{0}^{4}$ to be the average plaquette value, or to choose $u_{0}$ to be the average link value in the Landau gauge.

A natural question is how we should extend the mean field approximation if we employ smearing. One possibility is to express everything in terms of two quantities, $u_{0}$, a mean value for the unsmeared link, and $u_{S}$, a mean value for smeared links ${ }^{1}$. Applying the mean field approximation to SLiNC fermions we find

$$
\kappa_{c} \approx \frac{1}{8 u_{S}}, \quad c_{S W} \approx \frac{u_{S}}{u_{0}^{4}} .
$$

As a result, we find mean field improved expressions for $\kappa_{c}$ and $c_{S W}$ by performing the following replacements

$$
\kappa_{c}\left(g^{2}\right) \rightarrow \kappa_{c}^{M F}\left(g_{M F}^{2}, u_{S}\right)=\frac{u_{S}^{\mathrm{pert}}\left(g_{M F}^{2}\right)}{u_{S}} \kappa_{c}\left(g_{M F}^{2}\right)
$$

and

$$
c_{S W}\left(g^{2}\right) \rightarrow c_{S W}^{M F}\left(g_{M F}^{2}, u_{S}, u_{0}\right)=\frac{u_{S}}{u_{0}^{4}} \frac{u_{0}^{\text {pert }}\left(g_{M F}^{2}\right)^{4}}{u_{S}^{\text {pert }}\left(g_{M F}^{2}\right)} c_{S W}\left(g_{M F}^{2}\right) .
$$

Here $u_{S}$ and $u_{0}$ are the measured smeared and unsmeared links at the given coupling and $u_{S}^{\text {pert }}$ and $u_{0}^{\text {pert }}$ denote the corresponding expressions in lattice perturbation theory.

\footnotetext{
${ }^{1}$ We would like to thank Colin Morningstar for conversations on this point.
} 
We will use $u_{S}^{\text {pert }}$ derived from the smeared perturbative plaquette $P_{S}$

$$
u_{S}^{\text {pert }} \equiv P_{S}^{1 / 4}
$$

To one-loop order we have

$$
u_{S}^{\text {pert }}(\omega)=1-\frac{g_{M F}^{2} C_{F}}{16 \pi^{2}} k_{S}(\omega),
$$

where the one-loop contribution $k_{S}$ is [5]

$$
k_{S}(\omega)=\pi^{2}\left(0.732525-11.394696 \omega+50.245225 \omega^{2}\right) .
$$

The unsmeared perturbative value for $u_{0}^{\text {pert }}$ is $u_{0}^{\text {pert }}=u_{S}^{\text {pert }}(0)$. Inserting the result (4.6) into the mean field expressions (4.2) and (4.3) we obtain

$$
\begin{aligned}
\kappa_{c}^{M F}= & \frac{1}{8 u_{S}}\left[1+g_{M F}^{2} C_{F}\left(-0.008053+0.0500781 \omega-0.471784 \omega^{2}\right)\right], \\
c_{S W}^{M F}= & \frac{u_{S}}{u_{0}^{4}}\left\{1+g_{M F}^{2}\left[C_{F}\left(-0.0211635+0.115961 \omega+0.685247 \omega^{2}\right)\right.\right. \\
& \left.\left.+N_{c}\left(0.013777+0.015905 \omega-0.321899 \omega^{2}\right)\right]\right\} .
\end{aligned}
$$

The mean field improved quantities are expressed in terms of the boosted coupling $g_{M F}^{2}=g^{2} / u_{0}^{4}$. In [5] it is shown that $g_{M F}^{2}$ is a good expansion parameter in the case of the Symanzik improved gauge action with SLiNC fermions: in one-loop it does not differ very much from the coupling $g_{\overline{M S}}^{2}$ in the $\overline{M S}$-scheme. Comparing (4.7) and (4.8) with (3.10) and (3.6) we find that the one-loop correction terms are indeed smaller than in the naive perturbative expressions. Therefore, the mean field approximation has improved the perturbative behaviour as expected.

\section{Renormalization of currents}

We consider the renormalization constants for the following local bilinear quark operators

$$
S=\bar{\psi} 1 \psi, \quad P=\bar{\psi} \gamma_{5} \psi, \quad V=\bar{\psi} \gamma_{\mu} \psi, \quad A=\bar{\psi} \gamma_{\mu} \gamma_{5} \psi
$$

The corresponding renormalization factor for an operator $O$ has the general form

$$
Z_{O}=1-\frac{g^{2} C_{F}}{16 \pi^{2}}\left(\gamma_{O} \log \left(a^{2} \mu^{2}\right)+\mathscr{B}_{O}(\omega)\right)+\mathscr{O}\left(g^{4}\right) .
$$

Applying the mean field improvement as discussed in the preceding section the Z-factor is obtained as

$$
\begin{aligned}
Z_{O}^{M F} & =u_{S}\left(1-\frac{g_{M F}^{2} C_{F}}{16 \pi^{2}}\left(\gamma_{O} \log \left(a^{2} \mu^{2}\right)+\mathscr{B}_{O}(\omega)-k_{S}(\omega)\right)+\mathscr{O}\left(g_{M F}^{4}\right)\right) \\
& \equiv u_{S}\left(1-\frac{g_{M F}^{2} C_{F}}{16 \pi^{2}}\left(\gamma_{O} \log \left(a^{2} \mu^{2}\right)+\mathscr{B}_{O}^{M F}(\omega)\right)+\mathscr{O}\left(g_{M F}^{4}\right)\right),
\end{aligned}
$$

where $k_{S}(\omega)$ is given in (4.6). We expect that stout smearing leads to a more continuum-like behavior. For the choice $a \approx 1 / \mu$ this means that the correction term $\mathscr{B}_{O}$ should become small in 
order to achieve $Z_{O} \approx 1$. In Table 5.4 we show the results for the local operators. It is obvious that due to smearing with the selected value $\omega=0.1[6]$ the one-loop correction is diminished essentially.

\begin{tabular}{r|r|c|r|r|r|r}
$\mathrm{O}$ & \multicolumn{1}{|c|}{$\gamma_{O}$} & $\mathscr{B}_{O}(\omega)$ & $\mathscr{B}_{O}(0)$ & $\mathscr{B}_{O}(0.1)$ & $\mathscr{B}_{O}^{M F}(0)$ & $\mathscr{B}_{O}^{M F}(0.1)$ \\
\hline$S$ & -3 & $15.075-168.341 \omega+242.254 \omega^{2}$ & 15.075 & 0.663 & 7.845 & -0.280 \\
$P$ & -3 & $19.150-267.462 \omega+1065.55 \omega^{2}$ & 19.150 & 3.059 & 11.920 & 2.117 \\
$V$ & 0 & $11.911-170.763 \omega+754.029 \omega^{2}$ & 11.911 & 2.375 & 4.681 & 1.432 \\
$A$ & 0 & $10.717-127.200 \omega+342.380 \omega^{2}$ & 10.716 & 1.420 & 3.487 & 0.478
\end{tabular}

In order to show the effect on the renormalization factors themselves we need the values for $u_{S}$ and $u_{0}$. For $\beta=5.5$ we have $u_{S}=0.9404$ and $u_{0}=0.8495$ [12]. The following table shows the corresponding results for $a=1 / \mu$ and this selected $\beta$-value

\begin{tabular}{c|c|c|c}
$\mathrm{O}$ & $Z_{O}(\omega=0)$ & $Z_{O}(\omega=0.1)$ & $Z_{O}^{M F}(\omega=0.1)$ \\
\hline$S$ & 0.768 & 0.990 & 0.948 \\
$P$ & 0.706 & 0.953 & 0.882 \\
$V$ & 0.817 & 0.964 & 0.901 \\
$A$ & 0.836 & 0.978 & 0.927
\end{tabular}

In (5.5) we see that smearing shifts the renormalization factors towards unity showing a better continuum-like behaviour as promised.

\section{References}

[1] K. Symanzik, Nucl. Phys. B 226, 187 (1983).

[2] M. Lüscher and P. Weisz, Commun. Math. Phys. 97, 59 (1985) [Erratum-ibid. 98, 433 (1985)].

[3] B. Sheikholeslami and R. Wohlert, Nucl. Phys. B 259 (1985) 572.

[4] R. Horsley, H. Perlt, A. Schiller, P. E. L. Rakow and G. Schierholz, PoS LAT2007 (2007) 250 [arXiv:0710.0990 [hep-lat]].

[5] R. Horsley, H. Perlt, P. E. L. Rakow, G. Schierholz and A. Schiller, [arXiv:0807.0345 [hep-lat]].

[6] R. Horsley, Lattice 2008 Conference Proceedings.

[7] C. Morningstar and M. J. Peardon, Phys. Rev. D 69, 054501 (2004) [arXiv:hep-lat/0311018].

[8] R. Wohlert, DESY preprint 87/069 (unpublished).

[9] M. Lüscher and P. Weisz, Nucl. Phys. B 479, 429 (1996) [arXiv:hep-lat/9606016].

[10] S. Aoki and Y. Kuramashi, Phys. Rev. D 68, 094019 (2003) [arXiv:hep-lat/0306015].

[11] G. Martinelli, G. C. Rossi, C. T. Sachrajda, S. R. Sharpe, M. Talevi and M. Testa, Nucl. Phys. B 611 (2001) 311 [arXiv:hep-lat/0106003].

[12] QCDSF collaboration, in preparation. 